\title{
Polynomial Spline Collocation Methods for Volterra Integrodifferential Equations with Weakly Singular Kernels
}

\author{
Hermann BrunNer \\ Institut de Mathématiques, Université de Fribourg, CH-1700 Fribourg, \\ Switzerland
}

[Received 23 August 1984 and in revised form 14 January 1985]

In this paper we investigate the attainable order of (global) convergence of collocation approximations in certain polynomial spline spaces for solution of Volterra integrodifferential equations with weakly singular kernels. While the use of quasi-uniform meshes leads, due to the nonsmooth nature of these solutions, to convergence of order less than one, regardless of the degree of the approximating spline function, collocation on suitably graded meshes will be shown to yield optimal convergence rates.

\section{Introduction}

WE present a study of the convergence behaviour of collocation approximations in certain polynomial spline spaces for the solution of the Volterra integrodifferential equation

$$
y^{\prime}(t)=f[t, y(t)]+\int_{0}^{t}(t-s)^{-\alpha} k[t, s, y(s)] \mathrm{d} s, \quad t \in I:=[0, T],
$$

with $0<\alpha<1$ and $I$ compact, and with given initial condition $y(0)=y_{0}$. For ease of exposition we shall frequently employ the linear counterpart of (1.1),

$$
y^{\prime}(t)=a(t) y(t)+b(t)+\int_{0}^{t}(t-s)^{-\alpha} K(t, s) y(s) \mathrm{d} s, \quad t \in I
$$

in the analysis of the principal properties of these collocation approximations.

It will always be assumed that (1.1) and (1.2) possess unique solutions $y \in \mathrm{C}^{1}(\mathrm{I})$. Thus, in the case of equation (1.2), the given functions will be subject to the conditions $a, b \in \mathrm{C}(l)$ and $K \in \mathrm{C}(S)$, where $S:=\{(t, s): 0 \leqslant s \leqslant t \leqslant T\}$. In (1.1), the roles of $a(t)$ and $K(t, s)$ will be assumed by the partial derivatives $\partial f(t, y) / \partial y$ and $\partial k(t, s, y) / \partial y$, with the argument $y$ lying in some suitable neighbourhood of the exact solution. More precise conditions will be stated later.

For given $N \in \mathbb{N}$, let $\Pi_{N}:=\left\{t_{0}, \ldots, t_{N}\right\}\left(0=t_{0}<t_{1}<\cdots<t_{N}=T\right)$ denote a partition of the interval $I$ (for ease of notation we suppress the index $N$ in $t_{n}=t_{n}^{(N)}$ indicating the dependence of the mesh points on $N)$, and set $Z_{N}:=\left\{t_{n}: 1 \leqslant n \leqslant\right.$ $N-1\}$ (the set of interior mesh points), $\Sigma_{n}:=\left[t_{n}, t_{n+1}\right]$, and $h_{n}:=t_{n+1}-t_{n}(n=$ $0, \ldots, N-1$ ). We shall approximate the solution of $(1.1)$ (or of $(1.2)$ ) in the polynomial spline space

$$
S_{m}^{(0)}\left(Z_{N}\right):=\left\{u \in \mathrm{C}(I): u_{n} \in \pi_{m}\left(\Sigma_{n}\right)(n=0, \ldots, N-1)\right\},
$$


where $u_{n}=u \uparrow \Sigma_{n}$ and $\pi_{m}\left(\Sigma_{n}\right)$ is the space of real polynomial functions on $\Sigma_{n}$ of degree $\leqslant m$. We do this by seeking to determine an element $u$ in this space so that

$$
u_{n}^{\prime}(t)=f\left[t, u_{n}(t)\right]+\int_{0}^{t}(t-s)^{-\alpha} k[t, s, u(s)] \mathrm{d} s
$$

holds for all $t \in X_{n}(n=0, \ldots, N-1)$ with $X_{n}$ defined by

$$
X_{n}:=\left\{t_{n j}:=t_{n}+c_{j} h_{n}: 0 \leqslant c_{1}<\cdots<c_{m} \leqslant 1\right\} \subset \Sigma_{n} .
$$

The set $X(N):=\bigcup_{n=0}^{N-1} X_{n}$ will be referred to as the set of collocation points, which is completely characterized by the given partition $\Pi_{N}$ and by the collocation parameters $\left\{c_{i}\right\}_{i=1}^{m}$. The collocation equation (1.3), together with the initial condition $u_{0}\left(t_{0}\right)=y_{0}$, will define a unique approximation $u \in S_{m}^{(0)}\left(Z_{N}\right)$ whenever the mesh diameter,

$$
h\left(=h^{(N)}\right):=\max _{0 \leqslant n \leqslant N-1} h_{n},
$$

is sufficiently small; due to the nature of the problem (1.1), this approximation $u$ will be generated recursively by successive computation of its restrictions, $u_{0}, \ldots, u_{N-1}$ to the subintervals $\Sigma_{0}, \ldots, \Sigma_{N-1}$ given by $\Pi_{N}$.

In order to render the collocation equation (1.3) into a form which more clearly exhibits the recursive nature of the method, let $t=t_{n j}$ and define (using an obvious change of variable in the integrals over the subintervals $\Sigma_{i}$ )

$$
\begin{aligned}
& \Phi_{n i}^{(j)}\left[u_{i} ; \alpha\right]:= \\
& \int_{0}^{1}\left(\frac{t_{n j}-t_{i}}{h_{i}}-v\right)^{-\alpha} k\left[t_{n j}, t_{i}+v h_{i}, u_{i}\left(t_{i}+v h_{i}\right)\right] \mathrm{d} v \quad \text { if } 0 \leqslant i \leqslant n-1, \\
& \int_{0}^{c_{j}}\left(c_{j}-v\right)^{-\alpha} k\left[t_{n j}, t_{n}+v h_{n}, u_{n}\left(t_{n}+v h_{n}\right)\right] \mathrm{d} v \text { if } i=n, \quad(j=1, \ldots, m) .
\end{aligned}
$$

With this notation, (1.3) may be written in the form

$$
\begin{aligned}
u_{n}^{\prime}\left(t_{n j}\right)=f\left[t_{n j}, u_{n}\left(t_{n j}\right)\right]+h_{n}^{1-\alpha} \Phi_{n n}^{(j)}\left[u_{n} ; \alpha\right] & \\
& +\sum_{i=0}^{n-1} h_{i}^{1-\alpha} \Phi_{n i}^{(j)}\left[u_{i} ; \alpha\right] \quad(j=1, \ldots, m ; n=0, \ldots, N-1),
\end{aligned}
$$

where $u_{n}^{\prime}$ (which is an element of $\pi_{m-1}\left(\Sigma_{n}\right)$ ) may be written as

$$
u_{n}^{\prime}\left(t_{n}+v h_{n}\right)=\sum_{k=1}^{m} L_{k}(v) Y_{n k}, \quad t_{n}+v h_{n} \in \Sigma_{n},
$$

with $L_{k}(v)$ denoting $k$ th Lagrange fundamental polynomial associated with the collocation parameters $\left\{c_{j}\right\}_{j=1}^{m}$, and with $Y_{n k}:=u_{n}^{\prime}\left(t_{n}+c_{k} h_{n}\right)$. The corresponding representation for $u_{n}$ is thus given by

$$
u_{n}\left(t_{n}+v h_{n}\right)=y_{n}+h_{n} \sum_{k=1}^{m} a_{k}(v) Y_{n k} \quad \text { with } t_{n}+v h_{n} \in \Sigma_{n}
$$


here, we have set $y_{n}:=u_{n}\left(t_{n}\right)$, and

$$
a_{k}(v):=\int_{0}^{v} L_{k}(\tau) \mathrm{d} \tau \quad \text { with } v \in[0,1] \quad(k=1, \ldots, m)
$$

We observe that, for each $n=0, \ldots, N-1$, equation (1.5) represents a nonlinear system in $\mathbb{R}^{m}$ for the vector $Y_{n}:=\left[Y_{n 1}, \ldots, Y_{n m}\right]^{\top}$; once its components have been found, the collocation approximation and its derivative on the subinterval $\Sigma_{n}$ can be computed by the interpolation formulas (1.6b) and (1.6a). It is, incidentally, easily seen that if $f$ and $k$ in (1.1) are continuous functions, and if the collocation parameters are chosen so that $0=c_{1}<\cdots<c_{m}=1$, then the resulting collocation approximation $u$ is an element of the smoother polynomial spline space

$$
S_{m}^{(1)}\left(Z_{N}\right):=S_{m}^{(0)}\left(Z_{N}\right) \cap C^{1}(I) .
$$

In the following we shall study the attainable (global) order of convergence of the collocation approximation $u$ on $I$. To put our results into proper perspective, we briefly summarize some convergence results relating to special cases of (1.1).

(i) If $k(t, s, y) \equiv 0$, then (1.5) reduces to an $m$-stage implicit Runge-Kutta method for the initial-value problem: $y^{\prime}=f(t, y)$ with $y(0)=y_{0}$. (Compare, e.g., Guillou \& Soulé, 1969; Nørsett \& Wanner, 1979; also Callender, 1971.) It follows for $y \in \mathrm{C}^{m+1}(I)$ that

$$
\left\|y^{(\lambda)}-u^{(\lambda)}\right\|_{\infty}=O\left(N^{-m}\right) \quad(\lambda=0,1)
$$

on any uniform mesh $Y_{N}$.

(ii) This optimal convergence result remains valid for nonvanishing (smooth) kernels $k(t, s, y)$ and $\alpha=0$, provided $y \in \mathrm{C}^{m+1}(I)$ (see Brunner, 1973, 1981, 1984).

(iii) It has been shown (Lubich, 1983; Brunner, 1983) that the solution $y$ of the initial-value problem (1.1) with $0<\alpha<1$, and with smooth $k(t, s, y)$, lies in $\mathrm{C}^{m+1}(I)$ only in certain exceptional circumstances; if this is the case, then (1.8) will hold (compare Abdalkhani, 1982).

Typically, the solution of (1.1) corresponding to smooth $k(t, s, y)$ satisfies

$$
y \in \mathrm{C}^{1}(I) \text { with } y^{\prime} \in \operatorname{Lip}_{1-\alpha}(I) \text {. }
$$

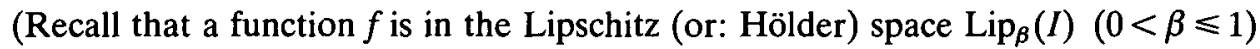
if, for all $t_{1}, t_{2} \in I$, there exists a constant $L$ so that

$$
\left|f\left(t_{1}\right)-f\left(t_{2}\right)\right| \leqslant L\left|t_{1}-t_{2}\right|^{\beta} .
$$

Such a function is frequently called Hölder-continuous, with Hölder exponent $\beta$.)

It thus follows from classical approximation theory (see, e.g. Timan, 1963; Powell, 1981) that (1.8) can no longer hold on uniform (or on quasi-uniform) meshes $\Pi_{N}$. In Sections 2 and 3 we shall show that, for such meshes, we can do no better than

$$
\left\|y^{(\lambda)}-u^{(\lambda)}\right\|_{\infty}=O\left(N^{-(1-\alpha)}\right) \quad(\lambda=0,1),
$$

independent of how we choose the degree $m$ of the approximation polynomial 
spline function $u \in S_{m}^{(0)}\left(Z_{N}\right)$. This is, in view of the results of Rice (1969) (see also de Boor, 1978), not surprising; however, the proof of (1.9) will not make use of these results.

In order to obtain collocation approximations possessing the optimal convergence behaviour described in $(1.8)$, two avenues are open to us:

(a) collocation in the polynomial spline space $S_{m}^{(0)}\left(Z_{N}\right)$, with $\Pi_{N}$ being a suitably graded mesh; or

(b) collocation in special nonpolynomial spline spaces (reflecting the nonsmooth behaviour of the solution of (1.1)), with uniform (or quasiuniform) mesh $\Pi_{N}$.

The latter approach has been analysed in Brunner (1983). In the present paper we shall deal with polynomial spline collocation on graded meshes: we shall give detailed proofs of the results announced in Brunner (1985 Section 5), using techniques introduced in connection with polynomial spline collocation on graded meshes for second-kind Volterra integral equations with weakly singular kernels.

The paper is organized as follows. Section 2 contains the convergence results, both for quasi-uniform and for graded meshes; their proofs will be given in Section 3. Since the integrals (1.4) occurring in the collocation equation (1.5) cannot, in general, be evaluated analytically, an additional discretization step will be required, involving the approximation of these integrals by appropriate (product) quadrature processes; this will be considered in Section 4. Finally, in Section 5 we present some numerical illustrations.

\section{The attainable order of global convergence}

With a given partition $\Pi_{N}$ of $I$ we associate the quantities

$$
h:=\max _{n} h_{n}, \quad h^{\prime}:=\min _{n} h_{n},
$$

where $h_{n}:=t_{n+1}-t_{n}(n=0, \ldots, N-1)$. Here, $h$ is the mesh diameter (for ease of notation we again suppress the index indicating the dependence of $h$ on $N$ ). A sequence of partitions (or meshes) for $I$ is called quasi-uniform if there exists a constant $\gamma$ independent of $N$ so that

$$
h / h^{\prime} \leqslant \gamma \text { uniformly for } N \in \mathbb{N} \text {. }
$$

If $\gamma=1$, then $\Pi_{N}$ is uniform, with $h=T N^{-1}$.

If the mesh points $\left\{t_{n}\right\}_{n=0}^{N}$ are given by

$$
t_{n}:=\left(\frac{n}{N}\right)^{r} T \quad(n=0, \ldots, N)
$$

then $\Pi_{N}$ is called a graded mesh; in the present context the so-called grading exponent $r \in \mathbb{R}$ will always satisfy $r \geqslant 1$. For $r=1$ we obtain again the uniform mesh.

THEOREM 2.1 Let the functions $a, b$, and $K$ in (1.2) be m-times continuously differentiable on their domains $I$ and $S$, respectively, and assume that $b$ and $K$ do 
not vanish identically. If $u \in S_{m}^{(0)}\left(Z_{n}\right)$ denotes the collocation approximation determined by (1.5), and if the underlying mesh sequence $\left(\Pi_{N}\right)$ is quasi-uniform, then, for all collocation parameters $\left\{c_{j}\right\}_{j=1}^{m}$ with $0 \leqslant c_{1}<\cdots<c_{m} \leqslant 1$, the resulting error $e:=y-u$ satisfies

$$
\left\|e^{(\lambda)}\right\|_{\infty}=O\left(N^{-(1-\alpha)}\right) \quad(\lambda=0,1) ;
$$

this estimate is best-possible in the sense that the exponent $1-\alpha$ cannot be replaced by some $\beta$ with $\beta>1-\alpha$.

The motivation for using graded meshes in the approximation by polynomial spline functions of nonsmooth functions of the form $f(t)=t^{\beta}(\beta>0)$ on $[0,1]$ was given by Rice (1969) (see also de Boor, 1978 (pp. 44-47, 189-191), Powell, 1981 (pp. 254-257)). This idea was subsequently adapted to generate high-order Galerkin, product integration, and collocation approximations by splines to the solutions of second-kind Fredholm integral equations with weakly singular kernels (compare, e.g. Chandler, 1979; Schneider, 1981, Vainikko \& Uba, 1981; Graham, 1982); and Vainikko, Pedas, \& Uba, 1984). Collocation methods for second-kind Volterra integral equations with weakly singular kernels using graded meshes are studied in Brunner (1985).

The following theorem shows that the use of suitably graded meshes in polynomial spline collocation for Volterra integrodifferential equations leads again to the optimal order of convergence (1.8) encountered when solving such equations with $\alpha=0$ on quasi-uniform meshes.

THeOREM 2.2 Let $a, b$ and $K$ in (1.2) be subject to the conditions stated in Theorem 2.1. If $u \in S_{m}^{(0)}\left(Z_{N}\right)$ is the collocation approximation defined by (1.5), and if the underlying mesh sequence $\left(\Pi_{N}\right)$ consists of graded meshes of the form (2.2), with grading exponent $r$ given by

$$
r=m /(1-\alpha)
$$

then, for all collocation parameters $\left\{c_{j}\right\}_{j=1}^{m}$ with $0 \leqslant<\cdots<c_{m} \leqslant 1$, the resulting error satisfies

$$
\left\|e^{(\lambda)}\right\|_{\infty}=O\left(N^{-m}\right) \quad(\lambda=0,1)
$$

Note that the grading exponent (2.4) is not determined by the solution $y$ itself-near $t=0$, it behaves like $y(t)=c t^{2-\alpha}+$ [smoother terms] (see Lubich, 1983; Brunner, 1983)-but by its derivative.

The proofs presented in the next section will show that the above convergence results remain valid for nonlinear Volterra integrodifferential equations (1.1): since the derivation of the equation satisfied by the collocation error will now involve a linearization step, the roles of $a(t)$ and $K(t, s)$ in (1.2) will then be assumed by the partial derivatives $f_{y}(t, y)$ and $k_{y}(t, s, y)$, and hence the smoothness requirements imposed on $a$ and on $K$ will have to be replaced by analogous smoothness (and boundedness) conditions for $f_{y}$ and $k_{y}$. 


\section{Proofs}

We first state a number of lemmas whose results will play a crucial role in the proofs of Theorems 2.1 and 2.2.

Lemma 3.1 Let $0<\alpha<1$, and assume that $0 \leqslant i<n \leqslant N-1$. Setting $t_{n j}:=$ $t_{n}+c_{j} h_{n}$ we have, for $j, l=l, \ldots, m$, and with $c_{j} \in[0,1]$,

$$
\int_{0}^{1}\left(\frac{t_{n j}-t_{i}}{h_{i}}-v\right)^{-\alpha} v^{l} \mathrm{~d} v<c(\alpha)(n-i)^{-\alpha}
$$

where the constant $c(\alpha)$ is given by

$$
c(\alpha):= \begin{cases}\gamma^{\alpha}(1+\gamma)^{\alpha} /(1-\alpha) & \text { if }\left(\Pi_{N}\right) \text { is quasi-uniform (cf. (2.1)); } \\ 2^{\alpha} /(1-\alpha) & \text { if }\left(\Pi_{N}\right) \text { is graded (cf. (2.2)), with } r>1 .\end{cases}
$$

Proof. See Brunner (1985) (Lemma 4.1, Lemma 4.4).

Lemma 3.2 Let the assumptions of Lemma 3.1 hold. Then the estimate

$$
\begin{aligned}
& \sum_{i=0}^{n-1} h_{i}^{1-\alpha} \int_{0}^{1}\left(\frac{t_{n j}-t_{i}}{h_{i}}-v\right)^{-\alpha} v^{l} \mathrm{~d} v \leqslant \frac{T^{1-\alpha}}{1-\alpha} \\
&(1 \leqslant n \leqslant N-1 ; j, l=1, \ldots,)
\end{aligned}
$$

is valid for quasi-uniform and graded mesh sequences $\left(\Pi_{N}\right)$.

Proof. By straightforward calculation using $t_{n j} \geqslant t_{n}$.

Lemma 3.3 Let $0<\alpha<1, \lambda \in\{0,1\}$, and $k \in\{0, \ldots, m+1\}$.

(i) If the mesh sequence $\left(\Pi_{N}\right)$ is quasi-uniform, then, for fixed $s \geqslant 1$,

$$
h_{i}^{m+\lambda} t_{i}^{t^{(2-\alpha)-k}}=\left\{\begin{array}{cc}
O\left(N^{-(m+\lambda)}\right) & \text { if } s(2-\alpha)-k \leqslant 0, \\
O\left(N^{-(1+\lambda-\alpha)}\right) & \text { if } s(2-\alpha)-k<0 \\
(i=1, \ldots, N) .
\end{array}\right.
$$

(ii) If the mesh sequence $\left(\Pi_{N}\right)$ is graded, and if the grading exponent $r$ is given by $r=m /(1-\alpha)$, then, for fixed $s \geqslant 1$,

$$
h_{i}^{m+\lambda} t_{i}^{(2-\alpha)-k}=O\left(N^{-(m+\lambda)}\right) \quad(i=1, \ldots, N)
$$

regardless of whether $s(2-\alpha)-k$ is positive or negative.

Proof. We first observe that the mesh diameters $h$ of quasi-uniform meshes satisfy, by (2.1) and by $h^{\prime} \leqslant T N^{-1}$,

$$
h \leqslant \gamma T N^{-1}=O\left(N^{-1}\right) .
$$

For graded meshes of the form (2.2), with $r>1$, we have

$$
h<r T N^{-1}=O\left(N^{-1}\right) \text {. }
$$

Hence, assertions (3.2a) and (3.2b) are trivial for $s(2-\alpha)-k>0$.

Suppose then that $s(2-\alpha)-k<0$. For quasi-uniform meshes we have

$$
t_{i} \geqslant t_{1}=h_{0} \geqslant h^{\prime} \geqslant h \gamma^{-1} \geqslant \gamma^{-1} T N^{-1} \quad(i=1, \ldots, N)
$$


and thus

$$
h_{i}^{m+\lambda} t_{i}^{s(2-\alpha)-k} \leqslant c N^{-[m+\lambda+s(2-\alpha)-k]} \leqslant c N^{-(\lambda+1-\alpha)}
$$

with

$$
c:=(\gamma T)^{m+\lambda}\left(\gamma^{-1} T\right)^{s(2-\alpha)-k} .
$$

The last part of $(3.2 \mathrm{a})$ is now a consequence of

$$
\dot{m}+\lambda+s(2-\alpha)-k \geqslant m+\lambda-(m+1)+s(2-\alpha) \geqslant \lambda-1+s-\alpha=\lambda+1-\alpha,
$$

since $k$ and $s$ satisfy, respectively, $0 \leqslant k \leqslant m+1$ and $s \geqslant 1$.

Consider now a sequence of graded meshes of the form (2.2), with $r>1$. Since $t_{i}=i^{r} T N^{-r}$, it follows that

$$
h_{i}=t_{i+1}-t_{i}=i^{r} T N^{-r}\left[\left(1+i^{-1}\right)^{r}-1\right]<r \cdot 2^{r-1} T i^{r-1} N^{-r}
$$

$(i=1, \ldots, N-1)$. We thus obtain, setting

$$
\begin{gathered}
c^{\prime}:=\max _{0 \leqslant k \leqslant m+1}\left(r \cdot 2^{r-1} T\right)^{m+\lambda} T^{s(2-\alpha)-k}, \\
h_{i}^{m+\lambda} t_{i}^{s(2-\alpha)-k}<c^{\prime} i^{r[s(2-\alpha)-(1-\lambda)]-(m+\lambda)} N^{-r[s(2-\alpha)-(1-\lambda)]}
\end{gathered}
$$

$(k=0, \ldots, m+1 ; s \geqslant 1)$. If $r=m /(1-\alpha)$, then the exponent of $i$ is easily seen to be nonnegative whenever $s \geqslant 1$. Hence, for $1 \leqslant i<N$ and $\lambda \in\{0,1\}$ we have

$$
h_{i}^{m+\lambda} t_{i}^{s(2-\alpha)-k}<c^{\prime} N^{-(m+\lambda)} \quad(k=0, \ldots, m+1) .
$$

We now return to the collocation equation (1.5): using (1.2) we see that the collocation error $e:=y-u$ satisfies

$$
\begin{aligned}
e_{n}^{\prime}\left(t_{n j}\right)=a\left(t_{n j}\right) e_{n}\left(t_{n j}\right)+h_{n}^{1-\alpha} & \int_{0}^{c_{i}}\left(c_{j}-v\right)^{-\alpha} K_{n j}\left(t_{n}+v h_{n}\right) e_{n}\left(t_{n}+v h_{n}\right) \mathrm{d} v \\
+ & \sum_{i=0}^{n-1} h_{i}^{1-\alpha} \int_{0}^{1}\left(\frac{t_{n j}-t_{i}}{h_{i}}-v\right)^{-\alpha} K_{n j}\left(t_{i}+v h_{i}\right) e_{i}\left(t_{i}+v h_{i}\right) \mathrm{d} v \\
& (j=1, \ldots, m ; n=0, \ldots, N-1),
\end{aligned}
$$

where $e_{n}$ denotes the restriction of $e$ to the subinterval $\Sigma_{n}$, with $e_{0}(0)=0$, and where we have set $K_{n j}(\cdot):=K\left(t_{n j}, \cdot\right)$.

It has been shown in Brunner (1983) that, under the hypotheses of Theorem 2.1, the exact solution of (1.2) may be expressed in the form of an absolutely and uniformly convergent infinite series whose terms involve products of integer powers of $t^{2-\alpha}$ and functions in $\mathrm{C}^{m+1}(I)$. If $\alpha$ is rational, $\alpha=p / q$, with $p$ and $q$ coprime (which, in view of practical applications and for ease of notation, we shall henceforth assume), then the solution may be written as

$$
y(t)=\sum_{s=0}^{q-1} w_{s}(t) t^{s(2-\alpha)}, \quad w_{s} \in C^{m+1}(I) \quad(s=0, \ldots, q-1) .
$$

Hence, on the first subinterval $\Sigma_{0}=\left[t_{0}, t_{1}\right]$ (where $t_{0}=0$ ) we have

$$
y\left(t_{0}+v h_{0}\right)=\sum_{l=0}^{m} c_{0 l} v^{l}+h_{0}^{2-\alpha} \mathrm{C}_{0}(v)+h_{0}^{m+1} R_{0}(v) \text { for } v \in[0,1]
$$


where we have set

$$
c_{0 l}:=\sum_{s=0}^{q-1} h_{0}^{s(2-\alpha)} c_{0 l}^{(s)}, \quad R_{0}(v):=\sum_{s=0}^{q-1} h_{0}^{s(2-\alpha)} R_{0 s}(v) v^{s(2-\alpha)},
$$

and

$$
C_{0}(v):=\sum_{s=1}^{q-1} h_{0}^{(s-1)(2-\alpha)}\left(v^{s(2-\alpha)}-1\right) \sum_{l=0}^{m} c_{0 l}^{(s)} v^{l}
$$

This follows from the application of Taylor's formula to the functions $\left\{w_{s}\left(t_{0}+\right.\right.$ $\left.\left.v h_{0}\right): v \in[0,1]\right\}$ in (3.4): the $c_{0 l}^{(s)}$ are the corresponding Taylor coefficients, and $R_{0 s}(v)$ denotes, except for the factor $h_{0}^{m+1}$, the remainder term. The term $h_{0}^{2-\alpha} C_{0}(v)$ in (3.5) clearly reflects the nonsmooth behaviour of $y$ at $t=0$.

Consider now a subinterval $\Sigma_{n}$ with $1 \leqslant n \leqslant N-1$; since $t_{n}>0$ we may write, again by Taylor's formula,

$$
y\left(t_{n}+v h_{n}\right)=\sum_{l=0}^{m} c_{n l} v^{l}+h_{n}^{m+1} R_{n}(v) \quad(v \in[0,1])
$$

where

$$
c_{n l}:=y^{(l)}\left(t_{n}\right) h_{n}^{l} / l !, \quad R_{n}(v):=\int_{0}^{v}(v-z)^{m} y^{(m+1)}\left(t_{n}+z h_{n}\right) \mathrm{d} z
$$

(note that, for $n \geqslant 1, y \in \mathrm{C}^{m+1}\left[t_{n}, T\right]$ ).

Expressions analogous to (3.5) and (3.6) can be derived for $y^{\prime}\left(t_{0}+v h_{0}\right)$ and $y^{\prime}\left(t_{n}+v h_{n}\right)(n \geqslant 1)$. Thus, setting

$$
u_{n}\left(t_{n}+v h_{n}\right):=\sum_{l=0}^{m} a_{n l} v^{l} \quad(v \in[0,1] ; \quad n=0, \ldots, N-1),
$$

and defining

$$
\beta_{n l}:=c_{n l}-\alpha_{n l} \quad(l=0, \ldots, m),
$$

we obtain the following expressions for the restrictions of the collocation error and its derivative to the subintervals $\Sigma_{n}$ :

$$
\begin{gathered}
e_{n}\left(t_{n}+v h_{n}\right)=\left\{\begin{array}{l}
\sum_{l=0}^{m} \beta_{0 l} v^{l}+h_{0}^{2-\alpha} C_{0}(v)+h_{0}^{m+1} R_{0}(v) \quad \text { if } n=0, \\
\sum_{l=0}^{m} \beta_{n l} v^{l}+h_{n}^{m+1} R_{n}(v) \text { if } 1 \leqslant n \leqslant N-1 ;
\end{array}\right. \\
e_{n}^{\prime}\left(t_{n}+v h_{n}\right)=\left\{\begin{array}{l}
h_{0}^{-1}\left(\sum_{l=1}^{m} l \beta_{0 l} v^{l-1}+h_{0}^{2-\alpha} C_{0}^{\prime}(v)+h_{0}^{m+1} R_{0}^{\prime}(v)\right) \quad \text { if } n=0, \\
h_{n}^{-1}\left(\sum_{l=1}^{m} l \beta_{n l} c^{l-1}+h_{n}^{m+1} R_{n}^{\prime}(v)\right) \text { if } 1 \leqslant n \leqslant N-1 .
\end{array}\right.
\end{gathered}
$$


Substitution of these error expressions in (3.3) leads to

$$
\begin{aligned}
\sum_{l=1}^{m}\left(l c_{j}^{l-1}-\right. & \left.h_{n} a_{n j} c_{j}^{l}-h_{n}^{2-\alpha} \int_{0}^{c_{j}}\left(c_{j}-v\right)^{-\alpha} K_{n j}\left(t_{n}+v h_{n}\right) v^{l} \mathrm{~d} v\right) \beta_{n l} \\
= & h_{n}\left(a_{n j}+h_{n}^{1-\alpha} \int_{0}^{c_{j}}\left(c_{j}-v\right)^{-\alpha} K_{n j}\left(t_{n}+v h_{n}\right) \mathrm{d} v\right) \beta_{n 0} \\
& +h_{n} \sum_{i=0}^{n-1}\left[h_{i}^{1-\alpha} \int_{0}^{1}\left(\frac{t_{n j}-t_{i}}{h_{i}}-v\right)^{-\alpha} K_{n j}\left(t_{i}+v h_{i}\right) \mathrm{d} v \cdot \beta_{i 0}\right] \\
& +h_{n} \sum_{i=0}^{n-1}\left[h_{i}^{1-\alpha} \sum_{l=1}^{m} \int_{0}^{1}\left(\frac{t_{n j}-t_{i}}{h_{i}}-v\right)^{-\alpha} K_{n j}\left(t_{i}+v h_{i}\right) v^{l} \mathrm{~d} v \cdot \beta_{i l}\right]+h_{n} r_{n j}
\end{aligned}
$$

$(j=1, \ldots, m ; n=1, \ldots, N-1)$ with $a_{n j}:=a\left(t_{n j}\right)$ and with

$$
\begin{aligned}
r_{n j}:= & -h_{n}^{m} R_{n}^{\prime}\left(c_{j}\right)+h_{n}^{m+1} a_{n j} R_{n}\left(c_{j}\right)+h_{n}^{1-\alpha} \int_{0}^{c_{j}}\left(c_{j}-v\right)^{-\alpha} K_{n j}\left(t_{n}+v h_{n}\right) h_{n}^{m+1} R_{n}(v) \mathrm{d} v \\
& +\sum_{i=1}^{n-1} h_{i}^{1-\alpha} \int_{0}^{1}\left(\frac{t_{n j}-t_{i}}{h_{i}}-v\right)^{-\alpha} K_{n j}\left(t_{i}+v h_{i}\right) h_{i}^{m+1} R_{i}(v) \mathrm{d} v \\
& +h_{0}^{1-\alpha} \int_{0}^{1}\left(\frac{t_{n j}-t_{0}}{h_{0}}-v\right)^{-\alpha} K_{n j}\left(t_{0}+v h_{0}\right)\left[h_{0}^{2-\alpha} C_{0}(v)+h_{0}^{m+1} R_{0}(v)\right] \mathrm{d} v
\end{aligned}
$$

$(n=1, \ldots, N-1)$. For $n=0$ we have

$$
\begin{aligned}
r_{0 j}:=h_{0}^{1-\alpha}\left(-C_{0}^{\prime}\left(c_{j}\right)\right. & -h_{0}^{m-1+\alpha} R_{0}^{\prime}\left(c_{j}\right)+h_{0} a_{0 j} C_{0}\left(c_{j}\right)+h_{0}^{m+\alpha} a_{0 j} R_{0}\left(c_{j}\right) \\
& \left.+\int_{0}^{c_{j}}\left(c_{j}-v\right)^{-\alpha} K_{0 j}\left(t_{0}+v h_{0}\right)\left[h_{0}^{2-\alpha} C_{0}(v)+h_{0}^{m+1} R_{0}(v)\right] \mathrm{d} v\right) .
\end{aligned}
$$

Since $e(t)$ is continuous at the knots $Z_{N}$ we have $e_{n}\left(t_{n}\right)=e_{n-1}\left(t_{n}\right)$ for all $t_{n} \in Z_{N}$. Thus, $C_{0}(1)=0$ and (3.7) imply

$$
\beta_{n 0}=\beta_{00}+\sum_{i=0}^{n-1} \sum_{l=1}^{m} \beta_{i l}+\sum_{i=0}^{n-1} h_{i}^{m+1} R_{i}(1) \quad(n=1, \ldots, N-1) .
$$

The initial value $e_{0}(0)=y(0)-u_{0}(0)=0$ furnishes the additional equation

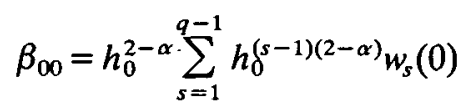

(cf. (3.5)). Hence it follows from $w_{s} \in \mathrm{C}^{m+1}(I)$ that there exists a finite constant $B_{0}$ so that

$$
\left|\beta_{00}\right| \leqslant h_{0}^{2-\alpha} B_{0}
$$

Combining this result with (3.11) we obtain

$$
\left|\beta_{n 0}\right| \leqslant \sum_{i=0}^{n-1}\left\|\boldsymbol{\beta}_{i}\right\|_{1}+h_{0}^{2-\alpha} B_{0}+\sum_{i=0}^{n-1} h_{i}^{m+1}\left|R_{i}(1)\right| \quad(n=1, \ldots, N-1),
$$

where we have introduced the vectors $\boldsymbol{\beta}_{i}:=\left[\beta_{i 1}, \ldots, \beta_{i m}\right]^{\top} \in \mathbb{R}^{m}$. We note for 
future reference that (3.9) (with $n=0),(3.10 \mathrm{~b}$ ), and (3.12) together imply that the initial vector $\boldsymbol{\beta}_{0}$ satisfies $\left\|\boldsymbol{\beta}_{0}\right\|_{1}=O\left(h_{0}^{2-\infty}\right)$.

We are now ready to rewrite (3.9) as a recurrence relation for these vectors, by introducing the matrices

$$
\begin{aligned}
& D_{n n}(\alpha):= \\
& {\left[l c_{j}^{l-1}-h_{n} a_{n j} c_{j}^{l}-h_{n}^{2-\alpha} \int_{0}^{c_{j}}\left(c_{j}-v\right)^{-\alpha} K_{n j}\left(t_{n}+v h_{n}\right) v^{l} \mathrm{~d} v: j, l=1, \ldots, m\right]} \\
& D_{n i}(\alpha):=\left[\int_{0}^{1}\left(\frac{t_{n j}-t_{i}}{h_{i}}-v\right)^{-\alpha} K_{n j}\left(t_{i}+v h_{i}\right) v^{l} \mathrm{~d} v: j, l=1, \ldots, m\right]
\end{aligned}
$$

$(0 \leqslant i \leqslant n-1)$ and the vectors $a_{n}:=\left[a_{n 1}, \ldots, a_{n m}\right]^{\top}$,

$$
\begin{gathered}
d_{n n}(\alpha):=\left[\int_{0}^{c_{j}}\left(c_{j}-v\right)^{-\alpha} K_{n j}\left(t_{n}+v h_{n}\right) \mathrm{d} v: j=1, \ldots, m\right]^{\top} \\
\boldsymbol{d}_{n i}(\alpha):=\left[\int_{0}^{1}\left(\frac{t_{n j}-t_{i}}{h_{i}}-v\right)^{-\alpha} K_{n j}\left(t_{i}+v h_{i}\right) \mathrm{d} v: j=1, \ldots, m\right]^{\top}
\end{gathered}
$$

$(0 \leqslant i \leqslant n-1)$, and

$$
r_{n}:=\left[r_{n 1}, \ldots, r_{n m}\right]^{\top},
$$

with $r_{n j}$ defined by (3.10). The desired recurrence relation assumes the form

$$
\begin{aligned}
& \boldsymbol{D}_{n n}(\alpha) \boldsymbol{\beta}_{n}= \\
& h_{n} \sum_{i=0}^{n-1} h_{i}^{1-\alpha} \boldsymbol{D}_{n i}(\alpha) \boldsymbol{\beta}_{i}+h_{n}\left(\left[a_{n}+h_{n}^{1-\alpha} d_{n n}(\alpha)\right] \beta_{n 0}+\sum_{i=0}^{n-1} h_{i}^{1-\alpha} d_{n i}(\alpha) \beta_{i 0}\right)+h_{n} r_{n} \\
& (n=0, \ldots, N-1),
\end{aligned}
$$

where the scalars $\beta_{i 0}$ are related to the vectors $\left\{\boldsymbol{\beta}_{i}\right\}_{i=0}^{N-1}$ by (3.11).

Observe first that, for all mesh sequences $\left(\Pi_{N}\right)$ possessing mesh diameters satisfying $h \downarrow 0$ as $N \rightarrow \infty$, the matrices $D_{n n}(\alpha)(n=0, \ldots, N-1)$ have uniformly bounded inverses for all sufficiently large values of $N$, since $a(t)$ and $K(t, s)$ are continuous (and hence bounded) on $I$ and $S$, and since

$$
\operatorname{det}\left[l c_{j}^{l-1}\right]=m ! \operatorname{det} V_{m} \neq 0
$$

here, $V_{m}$ denotes the Vandermonde matrix associated with the collocation parameters $\left\{c_{j}\right\}_{j=1}^{m}$. Thus, (3.16) (together with (3.11)) defines a unique sequence of vectors $\left(\boldsymbol{\beta}_{n}\right)$ whenever $\boldsymbol{h}$ is small enough; according to the remarks made at the beginning of the proof of Lemma 3.3, this holds both for quasi-uniform and for graded mesh sequences.

We shall now show that the 1 -norms (in $\mathbb{R}^{m}$ ) of these vectors $\boldsymbol{\beta}_{n}$ satisfy a generalized discrete Gronwall inequality. By (3.17) there exists a finite constant $d_{0}(\alpha)$ not depending on $N$, such that

$$
\left\|D_{n n}^{-1}(\alpha)\right\|_{1} \leqslant d_{0}(\alpha) \quad(n=0, \ldots, N-1)
$$


whenever $N$ is sufficiently large. Moreover, it follows readily from Lemma 3.1, (3.14), (3.15a,b), and from the continuity of the kernel $K(t, s)$, that

$$
\begin{array}{cc}
\left\|\boldsymbol{D}_{n i}(\alpha)\right\|_{1} \leqslant d_{1}(\alpha)(n-i)^{-\alpha} & (0 \leqslant i \leqslant n-1 \leqslant N-2), \\
\left\|\boldsymbol{d}_{n i}(\alpha)\right\|_{1} \leqslant d_{1}(\alpha)(n-i)^{-\alpha} & (0 \leqslant i \leqslant n-1 \leqslant N-2),
\end{array}
$$

where $d_{i}(\alpha)$ is some constant not depending on $N$ (but depending on $c(\alpha)$ defined in (3.1)), and

$$
\left\|d_{n n}(\alpha)\right\|_{1} \leqslant d_{2}(\alpha) \quad(n=0, \ldots, N-1) .
$$

Thus, multiplying (3.16) by $D_{n n}^{-1}(\alpha)$, taking 1-norms, and using (3.13), (3.12), we obtain the inequality

$$
\begin{aligned}
\left\|\boldsymbol{\beta}_{n}\right\|_{1} & \leqslant h\left(C_{0}^{\prime} h^{1-\alpha} \sum_{i=0}^{n-1}(n-i)^{-\alpha}\left\|\boldsymbol{\beta}_{i}\right\|_{1}+C_{1}^{\prime} \sum_{i=0}^{n-1}\left\|\boldsymbol{\beta}_{i}\right\|_{1}\right. \\
& \left.+C_{2}^{\prime} h^{1-\alpha} \sum_{i=1}^{n-1} \sum_{k=0}^{i-1}(n-i)^{-\alpha}\left\|\boldsymbol{\beta}_{k}\right\|_{1}\right) \\
& +h\left[h_{0}^{2-\alpha} B_{0}\left(C_{1}^{\prime}+C_{2}^{\prime} h^{1-\alpha} \sum_{i=0}^{n-1}(n-i)^{-\alpha}\right)+C_{1}^{\prime} \sum_{i=0}^{n-1} h_{i}^{m+1}\left|R_{i}(1)\right|\right. \\
& \left.+C_{2}^{\prime} h^{1-\alpha} \sum_{i=1}^{n-1} \sum_{k=0}^{i-1}(n-i)^{-\alpha} h_{k}^{m+1}\left|R_{k}(1)\right|\right]+h C_{1}\left\|\boldsymbol{r}_{n}\right\|_{1}
\end{aligned}
$$

( $n=0, \ldots, N-1)$ where $C_{0}^{\prime}, C_{1}, C_{1}^{\prime}$, and $C_{2}^{\prime}$ denote suitable constants not depending on $N$, but involving the constants $d_{i}(\alpha)(i=0,1,2)$.

The right-hand side of the above inequality may still be simplified, by using the discrete Dirichlet formula: we have, e.g.

$$
\begin{aligned}
\sum_{i=1}^{n-1} \sum_{k=0}^{i-1}(n-i)^{-\alpha}\left\|\boldsymbol{\beta}_{k}\right\|_{1} & =\sum_{i=0}^{n-2}\left(\sum_{k=i+1}^{n-1}(n-k)^{-\alpha}\right)\left\|\boldsymbol{\beta}_{i}\right\|_{1} \\
& \leqslant \sum_{i=0}^{n-1}\left(\sum_{k=i+1}^{n-1}(n-k)^{-\alpha}\right)\left\|\boldsymbol{\beta}_{i}\right\|_{1}
\end{aligned}
$$

setting $C_{0}:=\max \left(C_{0}^{\prime}, C_{1}^{\prime}, C_{2}^{\prime}\right)$, and using

$$
\begin{aligned}
1+h^{1-\alpha} \sum_{k=i}^{n-1}(n-k)^{-\alpha} & <1+h^{1-\alpha} \sum_{k=0}^{n-1}(n-k)^{-\alpha}<1+h^{1-\alpha} \int_{0}^{n} v^{-\alpha} \mathrm{d} v \\
& \leqslant 1+(N h)^{1-\alpha} /(1-\alpha),
\end{aligned}
$$

we obtain

$$
\left\|\boldsymbol{\beta}_{n}\right\|_{1} \leqslant h C_{0}\left(1+\frac{(N h)^{1-\alpha}}{1-\alpha}\right) \sum_{i=0}^{n-1}\left\|\boldsymbol{\beta}_{i}\right\|_{1}+h z_{n} \quad(n=1, \ldots, N-1),
$$

where, by the observation following (3.13), $\left\|\boldsymbol{\beta}_{0}\right\|_{1}=O\left(h_{0}^{2-q}\right)$, and where

$$
z:=h_{0}^{2-\alpha} C_{0} B_{0}\left(1+\frac{(N h)^{1-\alpha}}{1-\alpha}\right)+C_{0}\left(1+\frac{(N h)^{1-\alpha}}{1-\alpha}\right) \sum_{i=0}^{n-1} h_{i}^{m+1}\left|R_{i}(1)\right|+C_{1}\left\|r_{n}\right\|_{1} .
$$


It follows from the theory of discrete Gronwall inequalities (see McKee, 1982; Dixon \& McKee, 1983) that the order of $\left\|\boldsymbol{\beta}_{n}\right\|_{1}$ is governed by the order of the terms $h z_{n}$ in (3.18). In order to estimate these terms, note first that we have, by (2.1), $N h \leqslant \gamma T$ if $\left(\Pi_{N}\right)$ is quasi-uniform, and (by (2.2)) $N h<r T$ if $\left(\Pi_{N}\right)$ is graded. Thus, by (3.10a), the order of $z_{n}$ will be determined by the orders of the terms $h_{1}^{m+1}\left|R_{i}(v)\right|$ (with $v \in[0,1]$ ) and $h_{n}^{m}\left|R_{n}^{\prime}\left(c_{j}\right)\right|$. These Taylor remainder terms involve values of the $(m+1)$ th derivative of the exact solution $y$ of (1.2). By (3.4) we find, employing Leibniz's product rule,

$$
y^{(m+1)}(t)=w_{0}^{(m+1)}(t)+\sum_{s=1}^{q-1} \sum_{k=0}^{m+1} \gamma_{m k}^{(s)}(\alpha) w_{s}^{(m+1-k)}(t) t^{s(2-\alpha)-k} \text { for } t>0
$$

with

$$
\gamma_{m k}^{(s)}(\alpha):=\left(\begin{array}{c}
m+1 \\
k
\end{array}\right)\left(\begin{array}{c}
s(2-\alpha) \\
k
\end{array}\right) k !
$$

This reveals that the orders of the above Taylor remainder terms depend on the orders of the products $h_{i}^{m+\lambda} t_{i}^{s(2-\alpha)-k}(i=1, \ldots, N-1 ; k=0, \ldots, m+1)$, where $\lambda=0$ or $\lambda=1$, depending on whether we are dealing with expressions involving $R_{i}^{\prime}$ or $R_{i}$. Note also that it follows from the definition of the function $C_{0}(v)$ that $C_{0}(v)$ and $C_{0}^{\prime}(v)$ are both continuous and thus bounded on $[0,1]$. Hence, recalling Lemma 3.3 and Lemma 3.2 (which implies that the sums

$$
\sum_{i=1}^{n-1} h_{i}^{1-\alpha} \int_{0}^{1}\left(\frac{t_{n j}-t_{i}}{h_{i}}-v\right)^{-\alpha} \mathrm{d} v \quad(n=2, \ldots, N-1)
$$

are uniformly bounded by $T^{1-\alpha} /(1-\alpha)$ ), it is readily shown that, for $n=$ $1, \ldots, N-1$,

$$
h z_{n}= \begin{cases}O\left(N^{-(2-\alpha)}\right. & \text { if }\left(\Pi_{N}\right) \text { is quasi-uniform, } \\ O\left(N^{-(m+1)}\right. & \text { if }\left(\Pi_{N}\right) \text { is graded, with } r=m /(1-\alpha) .\end{cases}
$$

This, together with $\left\|\boldsymbol{\beta}_{0}\right\|_{1}=O\left(h_{0}^{2-\alpha}\right)$, yields

$$
\|\quad\| \boldsymbol{\beta}_{n} \|_{1}=O\left(h z_{n}\right)= \begin{cases}O\left(N^{-(2-\alpha)}\right) & \text { if }\left(\Pi_{N}\right) \text { is quasi-uniform, } \\ O\left(N^{-(m+1)}\right) & \text { if }\left(\Pi_{N}\right) \text { is graded, with } r=m /(1-\alpha) \\ & (n=1, \ldots, N-1) .\end{cases}
$$

Furthermore, (3.13) shows that, for quasi-uniform mesh sequences,

$$
\left|\beta_{n 0}\right|=N \cdot O\left(N^{-(2-\alpha)}\right)=O\left(N^{-(1-\alpha)}\right)
$$

by (3.7) and (3.8) this leads to

$$
\left|e_{n}^{(\lambda)}\left(t_{n}+v h_{n}\right)\right|=O\left(N^{-(1-\alpha)}\right) \quad(\lambda=0,1),
$$

uniformly for $t_{n}+v h_{n} \in \Sigma_{n}$ and $n=0, \ldots, N-1$ (as $N \rightarrow \infty$ with $N h \leqslant \gamma T$ ).

For graded mesh sequences with $r=m /(1-\alpha)$, (3.13) and (3.2b) imply $\left|\beta_{n 0}\right|=O\left(N^{-m}\right)$ (recall that, by (2.2), $h_{0}=T N^{-r}$, implying that $h_{0}^{1-\alpha}=O\left(N^{-m}\right)$ ). It then follows from (3.7) and (3.8) that

$$
\left|e_{n}^{(\lambda)}\left(t_{n}+v h_{n}\right)\right|=O\left(N^{-m}\right) \quad(\lambda=0,1),
$$


uniformly for $t_{n}+v h_{n} \in \Sigma_{n}$ and $n=0, \ldots, N-1$ (as $\left.N \stackrel{\circ}{\rightarrow} \infty\right)$. These two results are equivalent to (2.3) and (2.5).

So far it has been assumed that $\alpha$ is rational. If $\alpha$ is irrational, then, as has already been mentioned, the solution of (1.2) corresponding to functions $a, b$, and $K$ satisfying the hypotheses of Theorem 2.1 is of the form

$$
y(t)=\sum_{s=0}^{\infty} \psi_{s}(t) t^{s(2-\alpha)} \text { for } t \in I,
$$

with $\psi_{s} \in \mathrm{C}^{m+1}(I)$, and where the infinite series converges absolutely and uniformly on $I$ (Brunner, 1983); this also holds for (1.1), provided that the given functions $f$ and $k$ are subject to appropriate smoothness and boundedness conditions (Lubich, 1983). Hence, the above proofs are readily adapted to deal with this general situation; the key observation is, of course, the uniform convergence of the infinite series in (3.19) which implies, for example, that $y^{(m+1)}(t)(t>0)$ can be obtained by termwise differentiation of the right-hand side in (3.19).

Finally, we note that if the function $b$ in (1.2) is not smooth but has the form

$$
b(t):=b_{0}(t)+t^{1-\alpha} b_{1}(t) \text { with } b_{0}, b_{1} \in \mathrm{C}^{m}(I),
$$

then (Brunner, 1983) the corresponding solution is still of the form (3.19) (or, for $\alpha=p / q$, of the form (3.4)), with appropriately redefined functions $\psi_{s}$ (or $w_{s}$ ). Hence, as the above proofs show, the results of Theorems 2.1 and 2.2 cover this case as well (compare also the example in Section 5).

\section{Discretization of the collocation equation}

The collocation equation (1.5) cannot, in general, be used for the actual computation of the polynomial spline approximation $u \in S_{m}^{(0)}\left(Z_{N}\right)$ since the integrals (1.4) (with $u_{i}$ given by (1.6b)) can usually not be evaluated analytically. The fully discretized form of (1.5) is thus obtained by approximating these integrals by suitable product integration techniques. If we denote by $\hat{\Phi}_{n i}^{(i)}\left[u_{i} ; \alpha\right]$ the approximation to the integral $\Phi_{n i}^{(j)}\left[u_{i} ; \alpha\right]$, then the fully discretized version of $(1.5)$ is

$$
\begin{array}{r}
\hat{u}_{n}^{\prime}\left(t_{n j}\right)=f\left[t_{n j}, \hat{u}_{n}\left(t_{n j}\right)\right]+h_{n}^{1-\alpha} \hat{\Phi}_{n n}^{(j)}\left[\hat{u}_{n} ; \alpha\right]+\sum_{i=0}^{n-1} h_{i}^{1-\alpha} \hat{\Phi}_{n}^{(j)}\left[\hat{u}_{i} ; \alpha\right] \\
(j=1, \ldots, m ; n=0, \ldots, N-1) ;
\end{array}
$$

it defines, for all sufficiently small mesh diameters $h$, a polynomial spline function $\hat{u} \in S_{m}^{(0)}\left(Z_{N}\right)$ which, due to the errors induced by the numerical integration processes, will be different from the approximation $u \in S_{m}^{(0)}\left(Z_{N}\right)$ generated by the 'exact' collocation equation (1.5).

In the following we restrict the analysis to the case where the approximations $\hat{\Phi}_{n d}^{(i)}\left[\hat{u}_{i} ; \alpha\right]$ are of the form

$$
\hat{\Phi}_{n i}^{(j)}\left[\hat{u}_{i} ; \alpha\right]:=\left\{\begin{array}{l}
\sum_{l=1}^{m} w_{j l}^{(n, i)}(\alpha) k\left[t_{n j}, t_{i l}, \hat{u}_{i}\left(t_{i l}\right)\right] \quad \text { if } 0 \leqslant i \leqslant n-1, \\
\sum_{l=1}^{m} w_{j l}(\alpha) k\left[t_{n j}, t_{n}+c_{j} c_{l} h_{n}, \hat{u}_{n}\left(t_{n}+c_{j} c_{l} h_{n}\right)\right] \text { if } i=n
\end{array}\right.
$$


$(j=1, \ldots, m)$; here, the quadrature weights are given by

$$
\begin{gathered}
w_{j l}^{(n, i)}(\alpha):=\int_{0}^{1}\left(\frac{t_{n j}-t_{i}}{h_{i}}-v\right)^{-\alpha} L_{l}(v) \mathrm{d} v \quad(i \leqslant n-1), \\
w_{j l}(\alpha):=c_{j}^{1-\alpha} \int_{0}^{1}(1-v)^{-\alpha} L_{l}(v) \mathrm{d} v \quad(j, l=1, \ldots, m),
\end{gathered}
$$

and we have, in analogy to $(1.6 \mathrm{~b})$,

$$
\hat{u}_{n}\left(t_{n}+v h_{n}\right)=\hat{y}_{n}+h_{n} \sum_{k=1}^{m} a_{k}(v) \hat{Y}_{n k}, \quad t_{n}+v h_{n} \in \Sigma_{n},
$$

with $\hat{y}_{n}:=\hat{u}_{n}\left(t_{n}\right), \hat{Y}_{n k}:=\hat{u}_{n}^{\prime}\left(t_{n k}\right)$, and with $a_{k}(v)$ as in (1.7). Note that the quadrature approximations $\hat{\Phi}_{n n}^{(j)}\left[\hat{u}_{n} ; \alpha\right]$ employ only kernel values $k(t, s, y)$ with $s \leqslant t$.

Let $\varepsilon:=u-\hat{u}$, with the polynomial splines $u$ and $\hat{u}$ being given, respectively, by $(1.5)$ and $(4.1)$, where $u(0)=\hat{u}(0)=y(0)$. Since $\hat{u}$ induces the collocation error $\hat{e}:=y-\hat{u}$, which may be written as

$$
\hat{e}=(y-u)+(u-\hat{u})=e+\varepsilon,
$$

and since the attainable order of $e:=y-u$ is known by Theorems 2.1 and 2.2, the following result is not surprising.

THEOREM 4.1 Let the functions $a, b$, and $K$ in (1.2) be $m$ times continuously differentiable on their respective domains $I$ and $S$, and assume that $b$ and $K$ do not vanish identically. Moreover, let $\hat{u} \in S_{m}^{(0)}\left(Z_{N}\right)$ denote the solution of the fully discretized collocation equation (4.1), with quadrature approximations given by (4.2).

(i) If the underlying mesh sequence $\left(\Pi_{N}\right)$ is quasi-uniform, then $\hat{e}$ satisfies

$$
\left\|\hat{e}^{(\lambda)}\right\|_{\infty}=O\left(N^{-(1-\alpha)}\right) \quad(\lambda=0,1) .
$$

(ii) If $\left(\Pi_{N}\right)$ is the sequence of graded meshes (2.2), and if the grading exponent $r$ is $r=m /(1-\alpha)$, then we have

$$
\left\|\hat{e}^{(\lambda)}\right\|_{\infty}=O\left(N^{-m}\right) \quad(\lambda=0,1)
$$

These results hold for all collocation parameters $\left\{c_{j}\right\}_{j=1}^{m}$ with $0 \leqslant c_{1}<\cdots<c_{m} \leqslant 1$.

Proof. The techniques employed in the proofs of Theorems 2.1 and 2.2 (cf. Section 3) carry over, in a straightforward way, to the present situation. Here, the role of $e$ will be taken by $\varepsilon$, and instead of the Taylor remainder terms we now have to consider the quadrature errors

$$
E_{n i}^{(j)}\left[u_{i} ; \alpha\right]:=\Phi_{n i}^{(j)}\left[u_{i} ; \alpha\right]-\hat{\Phi}_{n i}^{(j)}\left[u_{i} ; \alpha\right] \quad(j=1, \ldots, m ; 0 \leqslant i \leqslant n \leqslant N-1) .
$$

Since the quadrature approximations (4.2) are of interpolatory type, it follows by the hypotheses of Theorem 4.1 that $E_{n i}^{(j)}\left[u_{i} ; \alpha\right]=O\left(N^{-m}\right)$. Moreover, it follows from Lemma 3.1 that the quadrature weights (4.3a) possess the property that

$$
\left|w_{j l}^{(n, i)}(\alpha)\right| \leqslant \text { const } \cdot(n-i)^{-\alpha} \quad(0 \leqslant i \leqslant n-1 ; j, l=1, \ldots, m) .
$$


Since $\varepsilon$ is an element of $S_{m}^{(0)}\left(Z_{N}\right)$, we have $\varepsilon_{n} \in \pi_{m}$. Moreover, the continuity conditions $\varepsilon_{n}\left(t_{n}\right)=\varepsilon_{n-1}\left(t_{n}\right)\left(t_{n} \in Z_{N}\right)$ lead to an inequality analogous to (3.13), with the roles of $\boldsymbol{\beta}_{i}$ and $\beta_{n 0}$ taken, respectively, by $\eta_{i}:=\left[\varepsilon_{i}\left(t_{i 1}\right), \ldots, \varepsilon_{i}\left(t_{i m}\right)\right]^{\top}$ and $\varepsilon_{n}\left(t_{n}\right)$. It is then easy to verify that the vectors $\eta_{n}$ satisfy a discrete Gronwall inequality of the form (3.18) where now the terms $z_{n}$ involve the quadrature errors $E_{n i}^{(j)}\left[u_{i} ; \alpha\right]$. These observations imply that

$$
\begin{aligned}
\left\|\eta_{n}\right\|_{1} & =O\left(N^{-(m+1)}\right) \quad(n=0, \ldots, N-1), \\
\left|\varepsilon_{n}\left(t_{n}\right)\right| & =O\left(N^{-m}\right) \quad(n=1, \ldots, N-1)
\end{aligned}
$$

(with $\varepsilon_{n}(0)=0$ ), leading to the estimate

$$
\|\varepsilon\|_{\infty}=O\left(N^{-m}\right)
$$

This result holds for quasi-uniform and graded mesh sequences since the functions $k\left[t_{n j}, s, u_{i}(s)\right]$ occurring in (1.4) are smooth for $s \in \Sigma_{i}$. We leave the details to the reader.

We mention in passing that the block-by-block methods of Makroglou (1981) (whose convergence was studied under the assumption that the exact solution of (1.1) be in $\mathrm{C}^{m+1}(I)$ ) can be viewed as fully discretized collocation methods, with the discretization based on the product integration formulas (4.2), (4.3).

\section{Example and numerical illustration}

Let the approximating polynomial spline space be $S_{2}^{(0)}\left(Z_{N}\right)$ with $m=2$. Setting $\hat{Y}_{n j}:=\hat{u}_{n}^{\prime}\left(t_{n j}\right)\left(j=1,2 ; 0 \leqslant c_{1}<c_{2} \leqslant 1\right)$, the corresponding fully discretized collocation equation (4.1) can be written as

$$
\hat{Y}_{n j}=f\left[t_{n j}, \hat{u}_{n}\left(t_{n j}\right)\right]+h_{n}^{1-\alpha} \hat{\Phi}_{n n}^{(j)}\left[\hat{u}_{n} ; \alpha\right]+\sum_{i=0}^{n-1} h_{i}^{1-\alpha} \hat{\Phi}_{n i}^{(j)}\left[\hat{u}_{i} ; \alpha\right]
$$

$(j=1,2 ; n=0, \ldots, N-1)$, with

$$
\hat{\Phi}_{n i}^{(j)}\left[\hat{u}_{i} ; \alpha\right]=\sum_{l=1}^{2} w_{j l}^{(n, i)}(\alpha) k\left[t_{n j}, t_{i l}, \hat{u}_{i}\left(t_{i l}\right)\right]
$$

$(j=1,2 ; 0 \leqslant i \leqslant n-1)$ and

$$
\hat{\Phi}_{n n}^{(j)}\left[\hat{u}_{n} ; \alpha\right]=\sum_{l=1}^{2} w_{j l}(\alpha) k\left[t_{n j}, t_{n}+c_{j} c_{l} h_{n}, \hat{u}_{n}\left(t_{n}+c_{j} c_{l} h_{n}\right)\right]
$$

$(j=1,2)$, where

$$
\hat{u}_{n}\left(t_{n}+v h_{n}\right)=\hat{y}_{n}+h_{n} \sum_{k=1}^{2} a_{k}(v) \hat{Y}_{n k}, \quad t_{n}+v h_{n} \in \Sigma_{n},
$$

with

$$
a_{1}(v)=\frac{1}{c_{2}-c_{1}} \int_{0}^{v}\left(c_{2}-\tau\right) \mathrm{d} \tau, \quad a_{2}(v)=\frac{1}{c_{2}-c_{1}} \int_{0}^{v}\left(\tau-c_{1}\right) \mathrm{d} \tau
$$


The quadrature weights in (5.2a) are given by (cf. (4.3a))

$$
\begin{aligned}
& w_{j 1}^{(n, i)}(\alpha)=\frac{1}{c_{2}-c_{1}} \int_{0}^{1}\left(\frac{t_{n j}-t_{i}}{h_{i}}-v\right)^{-\alpha}\left(c_{2}-v\right) \mathrm{d} v, \\
& w_{j 2}^{(n, i)}(\alpha)=\frac{1}{c_{2}-c_{1}} \int_{0}^{1}\left(\frac{t_{n j}-t_{i}}{h_{i}}-v\right)^{-\alpha}\left(v-c_{1}\right) \mathrm{d} v
\end{aligned}
$$

$(j=1,2 ; i \leqslant n-1)$, while those in (5.2b) have the form (cf. (4.3b))

$$
\begin{aligned}
& w_{j 1}(\alpha)=\frac{c_{j}^{1-\alpha}}{c_{2}-c_{1}} \int_{0}^{1}(1-v)^{-\alpha}\left(c_{2}-v\right) \mathrm{d} v, \\
& w_{j 2}(\alpha)=\frac{c_{j}^{1-\alpha}}{c_{2}-c_{1}} \int_{0}^{1}(1-v)^{-\alpha}\left(v-c_{1}\right) \mathrm{d} v
\end{aligned}
$$

$(j=1,2)$; they are easily computed analytically.

Once the values $\hat{Y}_{n 1}$ and $\hat{Y}_{n 2}$ have been determined by solving the (nonlinear) system (5.1), the collocation approximation $\hat{u} \in S_{2}^{(0)}\left(Z_{N}\right)$ for the subinterval $\Sigma_{n}$ is given by means of $(5.3)$, while its derivative is defined by

$$
\hat{u}_{n}^{\prime}\left(t_{n}+v h_{n}\right)=\frac{1}{c_{2}-c_{1}}\left[\left(c_{2}-v\right) \hat{Y}_{n 1}+\left(v-c_{1}\right) \hat{Y}_{n 2}\right] \text { for } t_{n}+v h_{n} \in \Sigma_{n} .
$$

In particular, we have (setting $v=1$ )

$$
\begin{gathered}
\hat{y}_{n+1}=\hat{y}_{n}+\frac{h_{n}}{2\left(c_{2}-c_{1}\right)}\left[\left(2 c_{2}-1\right) \hat{Y}_{n 1}+\left(1-2 c_{1}\right) \hat{Y}_{n 2}\right] \\
\hat{y}_{n+1}^{\prime}=\frac{1}{c_{2}-c_{1}}\left[\left(c_{2}-1\right) \hat{Y}_{n 1}+\left(1-c_{1}\right) \hat{Y}_{n 2}\right] .
\end{gathered}
$$

If we employ graded meshes of the form

$$
t_{n}=\left(\frac{n}{N}\right)^{r} T \quad(n=0, \ldots, N) \text { with } \quad r=2 /(1-\alpha),
$$

then, by Theorem 4.1, we obtain global convergence of order 2:

$$
\left\|y^{(k)}-\hat{u}^{(k)}\right\|_{\infty}=O\left(N^{-2}\right) \quad(k=0,1) .
$$

In contrast, the use of uniform (or quasi-uniform) meshes yields only

$$
\left\|y^{(k)}-u^{(k)}\right\|_{\infty}=O\left(N^{-(1-\alpha)}\right) \quad(k=0,1) .
$$

In order to illustrate these results we consider the integrodifferential equation

$$
y^{\prime}(t)=a(t) y(t)+b(t)+\int_{0}^{1} \lambda(t-s)^{-\alpha} y(s) \mathrm{d} s, \quad y(0)=1, \quad t \in[0, T],
$$

with $a(t) \equiv-1$ and $\lambda=-1$, and with $b(t)$ chosen so that $y(t)=t^{2-\alpha}$.

In Table 1 we list the norms of the resulting collocation error and its derivative, both for graded meshes with $r=2 /(1-\alpha)$ and for uniform meshes (which 
TABLE 1

\begin{tabular}{|c|c|c|c|c|c|c|}
\hline$N$ & \multicolumn{2}{|c|}{$\|e\|_{\infty} \underset{\left(\alpha=\frac{1}{3}\right)}{ }\left\|e^{\prime}\right\|_{\infty}$} & \multicolumn{2}{|c|}{$\left(\alpha=\frac{1}{2}\right){ }^{\| \epsilon}$} & \multicolumn{2}{|c|}{$\|e\|_{\infty} \quad\left(\alpha=\frac{2}{3}\right)$} \\
\hline 5 & $\begin{array}{c}5.60 \mathrm{E}-04 \\
(1.15 \mathrm{E}-03)\end{array}$ & $\begin{array}{c}1.17 \mathrm{E}-02 \\
(4.37 \mathrm{E}-02)\end{array}$ & $\begin{array}{c}1.23 \mathrm{E}-03 \\
(1.99 \mathrm{E}-03)\end{array}$ & $\begin{array}{c}2.29 \mathrm{E}-02 \\
(7.46 \mathrm{E}-02)\end{array}$ & $\begin{array}{c}4.96 \mathrm{E}-03 \\
(2.67 \mathrm{E}-03)\end{array}$ & $\begin{array}{c}4.65 \mathrm{E}-02 \\
(9.98 \mathrm{E}-02)\end{array}$ \\
\hline 10 & $\begin{array}{c}9.53 E-05 \\
(3.74 E-04)\end{array}$ & $\begin{array}{c}2.97 \mathrm{E}-03 \\
(2.81 \mathrm{E}-02)\end{array}$ & $\begin{array}{c}2.38 \mathrm{E}-04 \\
(7.28 \mathrm{E}-04)\end{array}$ & $\begin{array}{c}5.93 \mathrm{E}-03 \\
(5.41 \mathrm{E}-02)\end{array}$ & $\begin{array}{c}1.04 \mathrm{E}-03 \\
(1.11 \mathrm{E}-03)\end{array}$ & $\begin{array}{c}1.34 \mathrm{E}-02 \\
(8.14 \mathrm{E}-02)\end{array}$ \\
\hline 20 & $\begin{array}{c}1.55 E-05 \\
(1.19 E-04)\end{array}$ & $\begin{array}{c}7.31 \mathrm{E}-04 \\
(1.79 \mathrm{E}-02)\end{array}$ & $\begin{array}{c}4.67 \mathrm{E}-05 \\
(2.61 \mathrm{E}-04)\end{array}$ & $\begin{array}{c}1.45 \mathrm{E}-03 \\
(3.87 \mathrm{E}-02)\end{array}$ & $\begin{array}{c}1.93 \mathrm{E}-04 \\
(4.52 \mathrm{E}-04)\end{array}$ & $\begin{array}{c}3.32 \mathrm{E}-03 \\
(6.57 \mathrm{E}-02)\end{array}$ \\
\hline
\end{tabular}

correspond to $r=1$ ); the values for the uniform meshes appear between parentheses. The collocation parameters are the Gauss points: $c_{1}=(3-\sqrt{ } 3) / 6$, $c_{2}=(3+\sqrt{ } 3) / 6$.

In Table 2 we exhibit the values of the minimum and maximum step-sizes (i.e. $h^{\prime}=h_{0}, h=h_{N-1} ; h$ is the mesh diameter) for the graded meshes employed in the above computations. These values also indicate the practical limitation of collocation on graded meshes as described in Theorem 2.2: for fixed values of $\alpha$ and $m$, the initial step-size, $h^{\prime}=T N^{-r}$, becomes small as $N$ increases, thus creating a potentially serious source of round-off error which may contaminate the subsequent recursive process (compare, however, the remark preceding Table 3).

TABLE 2

\begin{tabular}{rccccccc}
\hline \multicolumn{1}{c}{$N$} & $h_{\left(\alpha=\frac{1}{3}\right)}^{\prime}$ & \multicolumn{2}{c}{$h^{\prime}$} & \multicolumn{2}{c}{$h$} & $h^{\prime}$ & $h$ \\
& \multicolumn{2}{c}{$\left(\alpha=\frac{1}{2}\right)$} & \multicolumn{2}{c}{$h$} & \multicolumn{2}{c}{$\left(\alpha=\frac{2}{3}\right)$} \\
\hline 5 & $8.00 \mathrm{E}-03$ & 0.488 & & $1.60 \mathrm{E}-03$ & 0.590 & $6.40 \mathrm{E}-05$ & 0.738 \\
10 & $1.00 \mathrm{E}-03$ & 0.271 & $1.00 \mathrm{E}-04$ & 0.344 & $1.00 \mathrm{E}-06$ & 0.469 \\
20 & $1.25 \mathrm{E}-04$ & 0.143 & $6.25 \mathrm{E}-06$ & 0.185 & $1.56 \mathrm{E}-08$ & 0.265 \\
\hline
\end{tabular}

We conclude by remarking that in many practical applications one is more interested in generating an approximation $\hat{u}$ which is very accurate at, or near, the right endpoint $t=T$ of the interval of integration, and not so much in one which is close to the exact solution over the entire interval $[0, T]$. The results contained in Table 3 (which are typical for more complex problems, too) show

TABLE 3

\begin{tabular}{|c|c|c|c|c|c|c|}
\hline$N$ & \multicolumn{2}{|c|}{$\left(\alpha=\frac{1}{3}\right)$} & \multicolumn{2}{|c|}{$\left(\alpha=\frac{1}{2}\right)$} & \multicolumn{2}{|c|}{$\left(\alpha=\frac{2}{3}\right)$} \\
\hline 5 & $\begin{array}{c}3.11 \mathrm{E}-04 \\
(4.59 \mathrm{E}-05)\end{array}$ & $\begin{array}{c}1.17 \mathrm{E}-02 \\
(1.20 \mathrm{E}-03)\end{array}$ & $\begin{array}{c}1.23 E-03 \\
(4.38 E-05)\end{array}$ & $\begin{array}{c}2.29 \mathrm{E}-02 \\
(1.17 \mathrm{E}-03)\end{array}$ & $\begin{array}{c}4.96 \mathrm{E}-03 \\
(9.97 \mathrm{E}-06)\end{array}$ & $\begin{array}{c}4.65 \mathrm{E}-02 \\
(1.19 \mathrm{E}-03)\end{array}$ \\
\hline 10 & $\begin{array}{c}5.19 \mathrm{E}-05 \\
(8.31 \mathrm{E}-06)\end{array}$ & $\begin{array}{c}2.97 \text { E }-03 \\
(2.53 \text { E }-04)\end{array}$ & $\begin{array}{c}2.23 \mathrm{E}-04 \\
(1.12 \mathrm{E}-06)\end{array}$ & $\begin{array}{l}5.93 \mathrm{E}-03 \\
(2.12 \mathrm{E} 04)\end{array}$ & $\begin{array}{c}1.04 \mathrm{E}-03 \\
(2.73 \mathrm{E}-05)\end{array}$ & $\begin{array}{c}1.34 \mathrm{E}-02 \\
(2.19 \mathrm{E}-04)\end{array}$ \\
\hline 20 & $\begin{array}{c}8.57 E-06 \\
(1.79 E-06)\end{array}$ & $\begin{array}{c}7.31 \mathrm{E}-04 \\
(5.40 \mathrm{E}-05)\end{array}$ & $\begin{array}{c}3.98 \mathrm{E}-05 \\
(2.82 \mathrm{E}-06)\end{array}$ & $\begin{array}{c}1.45 \mathrm{E}-03 \\
(3.33 \mathrm{E}-05)\end{array}$ & $\begin{array}{c}1.93 \text { E - } 04 \\
(1.55 E-05)\end{array}$ & $\begin{array}{c}3.32 \mathrm{E}-03 \\
(3.56 \mathrm{E}-05)\end{array}$ \\
\hline
\end{tabular}


that the collocation approximation $\hat{u} \in S_{2}^{(0)}\left(Z_{N}\right)$ obtained by using uniform meshes is always significantly more accurate at $t=T$ than the one where the underlying meshes are the properly graded ones (i.e., corresponding to $r=2 /(1-\alpha)$ ). This seems to reflect the 'self-correcting' property often observed in polynomial splines whose first derivatives are allowed to be discontinuous at their knots.

As in Table 1, the values between parentheses correspond to uniform meshes, while those appearing first belong to graded meshes with grading exponent $r=2 /(1-\alpha)$; the value of $T$ is $T=1$.

\section{Acknowledgement}

The author is grateful to one of the editors for his constructive criticism which helped to improve the presentation of the paper.

\section{REFERENCES}

AbDalkHANI, J. 1982 Collocation and Runge-Kutta-type methods for Volterra integral equations with weakly singular kernels. Ph.D. Thesis, Dalhousie University, Halifax, N.S.

DE BOOR, C. 1978 A Practical Guide to Splines. New York: Springer-Verlag.

BRUNNER, H. 1973 On the numerical solution of nonlinear Volterra integrodifferential equations. BIT 13, 381-390.

BRUNNER, H. 1981 The application of the variation of constants formulas in the numerical analysis of integral and integro-differential equations. Utilitas Math. 19, 255-290.

BRUNNER, H. 1983 Nonpolynomial spline collocation for Volterra equations with weakly singular kernels. SIAM J. Numer. Anal. 20, 1106-1119.

Brunner, H. 1984 Implicit Runge-Kutta methods of optimal order for Volterra integro-differential equations. Math. Comp. 42, 95-109.

BRUNNer, H. 1985 The approximate solution of Volterra equations with nonsmooth solutions. Utilitas Math. 27, 57-95.

Callender, E. D. 1971 Single step methods and low order splines for solutions of ordinary differential equations. SIAM J. Numer. Anal. 8, 61-66.

Chandler, G. A. 1979 Superconvergence of numerical methods to second kind integral equations. Ph.D. Thesis, Australian National University, Canberra.

Dixon, J. \& McKeE, S. 1983 Singular Gronwall inequalities. Numerical Analysis Report NA/83/44, Hertford College, University of Oxford.

Graham, I. G. 1982 Galerkin methods for second kind integral equations with singularities. Math. Comp. 39, 519-533.

Guillou, A. \& Soulé, J. L. 1969 La résolution numérique des problèmes différentiels aux conditions initiales par des méthodes de collocation. R.A.I.R.O. 3, 17-44.

LuBich, CH. 1983 Runge-Kutta theory for Volterra and Abel integral equations of the second kind. Math. Comp. 41, 87-102.

MAKROGLOU, A. 1981 A block-by-block method for Volterra integro-differential equations with weakly-singular kernel. Math. Comp. 37, 95-99.

McKeE, S. 1982 Generalised discrete Gronwall lemmas. Z. Angew. Math. Mech. 62, 429-434.

NørsetT, S. P. \& Wanner, G. 1979 The real-pole sandwich for rational approximations and oscillation equations. BIT 19, 79-94.

Powell, M. J. D. 1981 Approximation Theory and Methods. Cambridge: Cambridge University Press.

RICE, J. R. 1969 On the degree of convergence of nonlinear spline approximation. In: Approximation with Special Emphasis on Spline Functions (I. J. Schoenberg, Ed.). New York: Academic Press, 349-365. 
SCHNeIDER, C. 1981 Product integration for weakly singular integral equations. Math. Comp. 36, 207-213.

Timan, A. F. 1963 Theory of Approximation of Functions of a Real Variable. Oxford: Pergamon Press.

VAINIKKo, G., PEDAs, A. \& UBA, P. 1984 Methods for Solving Weakly Singular Integral Equations (in Russian). Tartu State University.

VAINIKKo, G. \& UBA, P. 1981 A piecewise polynomial approximation to the solution of an integral equation with weakly singular kernel. J. Austral. Math. Soc. Ser. B. 22, 431-438. 